\title{
Combination treatment significantly enhances the efficacy of antitumor therapy by preferentially targeting angiogenesis
}

\author{
Pawan Kumar ${ }^{1}$, Ryan Benedict ${ }^{1}$, Fernando Urzua ${ }^{1}$, Claudia Fischbach ${ }^{2}$, David Mooney $^{2}$ \\ and Peter Polverini ${ }^{3}$ \\ ${ }^{1}$ Department of Biological and Material Sciences, University of Michigan School of Dentistry, Ann Arbor, \\ MI, USA; ${ }^{2}$ Division of Engineering and Applied Sciences, Harvard University, Cambridge, MA, USA \\ and ${ }^{3}$ Department of Oral Medicine, Pathology and Oncology, University of Michigan School of Dentistry, \\ Ann Arbor, MI, USA
}

\begin{abstract}
Radiotherapy is one of the most widely used cancer treatments, but it is often unsuccessful due to the development of radioresistance by tumor cells and endothelial cells (ECs) lining the tumor blood vessels. We have previously shown that ECs are protected against ionizing irradiation primarily via the activation of the phosphoinositide 3-kinase (PI3 K)-Akt-Bcl-2 survival pathway. Here we report that combination treatment with low doses of PI3 K inhibitor (LY294002), cisplatin and $\gamma$-irradiation resulted in significantly higher (61\%) EC death as compared to each agent used alone $(17,17$ and $11 \%$, respectively). This combination treatment was equally effective in inducing tumor cell death $(72 \%)$. Combination treatment also significantly inhibited EC tube formation in Matrigel (75\%) as compared to each of the agents used alone (8, 8 and $18 \%$ for LY294002, cisplatin and $\gamma$-irradiation, respectively). In our in vivo severe combined immunodeficient mouse model of human tumor growth and angiogenesis, combination treatment with low doses of LY294002, cisplatin and irradiation significantly inhibited the growth of human oral squamous carcinoma (OSCC-3) as well as prostate cancer (LnCap). The combination therapy was also very effective in inhibiting tumor angiogenesis where it showed a greater than $90 \%$ decrease in neovascularization. In contrast, combination treatment showed only a $29 \%$ inhibition of physiological angiogenesis. Taken together, these results suggest a potentially novel strategy to overcome the resistance in ECs lining tumor blood vessels, thereby enhancing the effectiveness of the radiation and chemotherapy. Moreover, this strategy of using a combination of low doses of PI3K/Akt inhibitor, cisplatin and radiation has the potential of significantly decreasing untoward side effects associated with the maximum tolerated doses of radiation and chemotherapy while maintaining their therapeutic efficacy.

Laboratory Investigation (2005) 85, 756-767, advance online publication, 2 May 2005; doi:10.1038/labinvest.3700272
\end{abstract}

Keywords: angiogenesis; VEGF; apoptosis; combination therapy; endothelial cells; PI3 K

Tumor angiogenesis is not only essential for tumor growth, ${ }^{1,2}$ it also plays a central role in tumor progression ${ }^{3}$ and metastasis. ${ }^{4}$ Tumor angiogenesis is tightly regulated by a local balance of proangiogenic and antiangiogenic factors. ${ }^{5}$ Vascular endothelial growth factor (VEGF), one of the most important angiogenic proteins is expressed by a number of human tumors. ${ }^{6,7}$ In addition to mediating angiogenesis, VEGF plays an important role in protecting

Correspondence: Dr P Polverini, DDS, DMSc, The University of Michigan School of Dentistry, 1011 N University Ave, Room \# 1234, Ann Arbor, MI 48109, USA.

E-mail: neovas@umich.edu

Received 8 October 2004; revised 14 February 2005; accepted 18 February 2005; published online 2 May 2005 endothelial cells (ECs) from the proapoptotic effects of ionizing radiation, chemotherapeutic agents, and antiangiogenic factors such as thrombospondin- $10^{8,9}$ Numerous efforts have been directed at developing strategies to use VEGF as a potential therapeutic target. ${ }^{10}$ Inhibition of VEGF-induced angiogenesis either by neutralizing antibodies or by dominant negative soluble receptor significantly enhanced radiation-mediated EC death and reduced the growth of primary as well as metastatic tumors., ${ }^{8,11}$ However, long-term experiments with anti-VEGF treatment revealed evidence of eventual relapse and progression of tumor growth, even if the treatment was continued. ${ }^{12}$ Therefore, targeting one such growth factor with a neutralizing antibody or dominant negative soluble receptor could be 
overcome by the emergence of tumor variants that induce angiogenesis via the production of other proangiogenic factors. ${ }^{13}$ Recent reports have suggested that combinational treatment with different antiangiogenesis agents $^{14,15}$ or antiangiogenesis agents combined with anticytotoxic agents ${ }^{12}$ may be more effective than single agent therapy.

Ionizing radiation has been one of the most widely used treatments for a wide variety of solid tumors and is thought to act by directly targeting clonogens. ${ }^{16}$ However, EC apoptosis is required for clonogenic cell dysfunction. ${ }^{17}$ ECs lining the tumor blood vessels are normally quite resistant to radiation therapy and require quite high doses of ionizing irradiation to induce apoptosis. ${ }^{18}$ One of the reasons for tumor EC radioresistance could be the presence of abundant growth factors, particularly VEGF in the tumor microenvironment. ${ }^{8}$ We have recently shown that VEGF protects ECs against ionizing radiation by activating the phosphionositide 3-kinase (PI3K)/Akt pathway which leads to the enhanced expression of the antiapoptotic protein, Bcl-2. ${ }^{19}$

The PI3K/Akt pathway phosphorylates several downstream targets, including Bad and caspase-9, thus inhibiting their proapoptotic functions. ${ }^{20,21} \mathrm{In}$ addition, many other members of the apoptotic machinery, as well as transcription factors, contain the Akt consensus phosphorylation site, ${ }^{22}$ further suggesting that the PI3K/Akt pathway plays a prominent role in inhibiting apoptosis. Therefore, targeting the PI3K/Akt, a key survival signaling pathway, may provide the highest degree of synergistic antitumor activity with traditional therapies. In the present study, we demonstrate that a combination of low doses of PI3 K inhibitor (LY294002) and cisplatin significantly enhances the therapeutic efficacy of radiation therapy by preferentially targeting tumor blood vessels.

\section{Materials and methods}

\section{Endothelial Cell (HDMEC) Culture}

HDMECs $\left(2 \times 10^{5}\right)$ were plated in $60 \mathrm{~mm}$ dishes coated with a thin layer of type I collagen gel and incubated overnight at $37^{\circ} \mathrm{C}$ in endothelial growth medium (EGM)-2 MV medium. ${ }^{19}$ HDMECs were then incubated in growth factor free endothelial basal medium (EBM)-2 medium supplemented with $5 \%$ FBS for $1 \mathrm{hr}$ prior to stimulation with $50 \mathrm{ng} / \mathrm{ml}$ of VEGF. After 3 days of culture with VEGF, HDMECs were exposed to different doses of PI3 K inhibitor (LY294002), chemotherapeutic agent (cisplatin) or $\gamma$-irradiation. At day 0,3 and 6 , five random high-power fields $(\times 200)$ from each sample group were photographed with a digital camera (Nikon Inc., Melville, NY, USA) and then counted using Metamorph (Universal Imaging Corporation, Downingtown, PA, USA). The proliferation index (PI) was calculated by dividing cell count at day 6 by the cell count at day 3 for each group. \% HDMEC survival for each was calculated: PI (Test group) / PI (VEGF) $\times 100$.

\section{Oral Squamous Carcinoma Cells (OSCC-3) Culture}

OSCC-3 were plated in $60 \mathrm{~mm}$ dishes and cultured overnight in DMEM supplemented with $10 \%$ serum. OSCC-3 cells were then exposed to different doses of PI3 K inhibitor (LY294002), chemotherapeutic agent (cisplatin) or $\gamma$-irradiation for 3 days. At day 0 and 3, five random high-power fields $(\times 200)$ from each sample group were photographed with a digital camera (Nikon Inc., Melville, NY, USA) and then counted using Metamorph (Universal Imaging Corporation, Downingtown, PA, USA). The proliferation index (PI) was calculated by dividing the cell count at day 3 by the cell count at day 0 for each group. \%OSCC-3 survival for each was calculated: PI (Test group)/PI (No-Treatment) × 100 .

\section{Matrigel In Vitro HDMEC Tube Formation Assay}

Matrigel (125 $\mu$ l, growth factor reduced), after thawing on ice, was plated in eight-well chamber slides. ${ }^{23}$ These slides were then incubated at $37^{\circ} \mathrm{C}$ for $30 \mathrm{~min}$ to allow the Matrigel to polymerize. HDMECs $\left(400 \mu \mathrm{l} ; 4 \times 10^{4}\right.$ cells/ml in EBM-2 media supplemented with $2 \%$ serum) were added to each well and treated with VEGF $(50 \mathrm{ng} / \mathrm{ml})$ in the presence or absence of PI3 K inhibitor (LY294002) or the chemotherapeutic agent (cisplatin). The chamber slides were then incubated for $16-18 \mathrm{~h}$ at $37^{\circ} \mathrm{C}$ in $5 \% \mathrm{CO}_{2}$ humidified atmosphere. At the end of incubation, the culture media was very carefully aspirated off the Matrigel surface and the cells were fixed with methanol and stained with Diff-Quick solution II. Each chamber was photographed with a digital camera (Nikon Inc., Melville, NY, USA) and total tube area in each chamber was calculated using Metamorph software (Universal Imaging Corporation, Downingtown, PA, USA) and expressed as an angiogenesis score.

\section{Preparation of PLG-Matrices}

A copolymer of D, L-lactide and glycolide (PLG, 85:15 molar ratio) was used in a gas foaming, particulate leaching process to form matrices for VEGF delivery as described before. ${ }^{24}$ In brief, granular PLG (3 mg) was mixed with $0.15 \mathrm{ml}$ of aqueous $0.1 \%$ alginate solution, and $0.1,0.25,0.5$ or $1 \mu \mathrm{g}$ of VEGF. The samples were lyophilized to form a powder, mixed with $1.5 \mathrm{mg}$ of microspheres and $57 \mathrm{mg}$ of $\mathrm{NaCl}$ and compression molded into disks, and foamed with high pressure $\mathrm{CO}_{2}$ gas (800 psi). The $\mathrm{NaCl}$ particles were leached from the matrices, and any alginate present was crosslinked, by immersing each matrix in $0.1 \mathrm{M} \mathrm{CaCl}_{2}$ for $10 \mathrm{~h}$. Matrices were sterilized by immersing in $100 \%$ 
ethanol for $15 \mathrm{~min}$ followed by washing them five times with sterile PBS.

\section{In Vivo Tumor Development Assay}

CB.17. Severe combined Immunodeficient (SCID) mice (Taconic, Germantown, NY, USA) received subcutaneous injections of $9 \times 10^{5}$ HDMEC and $1 \times 10^{5}$ OSCC-3 or LnCap cells, mixed with $100 \mu \mathrm{l}$ of Matrigel, into both rear hind limbs. After 6 days when the average tumor volume reached around $150 \mathrm{~mm}^{3}$, mice were stratified into groups (five mice per group), so that the mean tumor volume in each group was comparable. At days 6, 9 and 14, animals were treated with LY294002 $(5 \mathrm{mg} / \mathrm{kg})$ and/or Cisplatin $(5 \mathrm{mg} / \mathrm{kg})$ via I.P. injections. On day 7 , animals were anesthetized and placed in a specially designed tray in such a way that the hind limbs were positioned in the inner chamber and the rest of the body in the outer chamber that was shielded from irradiation by a lead shield. Tumor volume measurements began on day 1 (tumor inoculation) and continued twice a week until the tumor volumes exceeded $10 \%$ of the body size. Tumor volumes were calculated using the formula, volume $\left(\mathrm{mm}^{3}\right)=L \times W^{2} / 2$ (length $L, \mathrm{~mm}$; width $W, \mathrm{~mm}$ ). Length and width were measured using a digital caliper. Data were calculated as the percentage of the tumor volume at day 3 and graphed as folds change in tumor volume + s.e. for each treatment group.

\section{In Vivo Tumor Angiogenesis Assay}

The effect of combination therapy on tumor angiogenesis and normal angiogenesis at a distal site (in the same animals) was examined using our SCID mouse model..$^{25,26}$ Cells $\left(9 \times 10^{5}\right.$ HDMEC and $1 \times 10^{5}$ OSCC-3) were mixed with $100 \mu \mathrm{l}$ of Matrigel and injected into the rear hind limb of SCID mice (10 mice per group). For normal angiogenesis studies, HDMECs $\left(1 \times 10^{6}\right)$ were mixed with a 1:1 mixture of Matrigel and EGM2-MV and seeded onto PLGmatrices. These matrices were implanted subcutaneously into the dorsal region of SCID mice. At days 2, 5 and 10 animals were treated with LY294002 $(5 \mathrm{mg} / \mathrm{kg})$ and/or Cisplatin $(5 \mathrm{mg} / \mathrm{kg})$ via I.P. injections. On day 3 animals were irradiated with a single dose of $10 \mathrm{~Gy}$. After 14 days, animals were sacrificed; tumors were carefully retrieved, measured with calipers, and weighed on an electronic balance. Tumors and PLG scaffolds were fixed overnight with $10 \%$ phosphate-buffered formalin and paraffin embedded.

\section{Immunolocalization of Von Willebrand Factor and CD31}

Tissue sections were deparaffinized and antigen retrieval was achieved by pressure cooking in a
Decloaking chamber (Biocare Medical, Walnut Creek, CA, USA) at $120^{\circ} \mathrm{C}$ for $20 \mathrm{~min}$. For CD31 and Von Willebrand factor staining, tissue sections were treated with peroxide block solution for $5 \mathrm{~min}$ at room temperature followed by $30 \mathrm{~min}$ of incubation with primary antibody (anti-CD31 or anti-Von Willebrand factor) at room temperature. Slides were further incubated for $30 \mathrm{~min}$ with HRP labeled polymer (Dako EnVision + System, Kit) and developed with AEC + chromogen (Von Willebrand factor) or DAB chromogen (CD31). The number of stained microvessels were counted blindly in six random high-power fields per implant at $\times 200$ magnification. We also quantified the vessel area in the tumor as well as the scaffolds using Metamorph software. Six implants (tumors as well as scaffolds) were analyzed per condition.

\section{In Situ TUNEL Assay and Activated Caspase-3 Staining}

Tissue sections were deparaffinized and antigen retrieval was achieved as described above. These sections were stained for terminal deoxynucleotidyl transferase (tdT)-mediated dUTP-biotin nick end labeling (TUNEL)-positive cells using KLENOWFragEL DNA Fragment Detection Kit (Calbiochem, San Diego, CA, USA) according to the manufacturer's instructions. For activated caspase-3, staining tissue sections were incubated with rabbit monoclonal anticleaved caspase-3 antibody (Cell Signaling) for $30 \mathrm{~min}$ and developed with Dako EnVision + DAB system as described above.

\section{Results}

PI3K Inhibitor (LY294002), Chemotherapeutic Agent (Cisplatin), and $\gamma$-Irradiation Induces a Dose-Dependent Increase in Endothelial and Tumor Cell Death

ECs were cultured in $60 \mathrm{~mm}$ dishes on a thin layer of collagen, stimulated with VEGF for $72 \mathrm{~h}$ at $37^{\circ} \mathrm{C}$ and then exposed to different doses of LY294002, cisplatin, and $\gamma$-irradiation. The LY294002 treatment resulted in a dose-dependent decrease in human dermal microvascular endothelial cell (HDMEC) survival. At $1 \mu \mathrm{M}$ LY294002 resulted in an $18 \%$ inhibition of HDMEC survival which was not statistically significant (Figure 1a). However, LY294002 at 10 and $15 \mu \mathrm{M}$ significantly inhibited HDMEC survival (42 and $64 \%$, respectively). Similarly, cisplatin treatment resulted in a dose-dependent decrease in HDMEC survival with 18, 30 and $52 \%$ inhibition of HDMEC survival at $0.5,1$ and $5 \mu \mathrm{M}$ doses, respectively (Figure 1b). $\gamma$-irradiation treatment showed 8 and 18\% inhibition of HDMEC survival at 5 and $10 \mathrm{~Gy}$ doses, respectively (Figure 1c). However, $\gamma$-irradiation treatment at a $20 \mathrm{~Gy}$ dose 

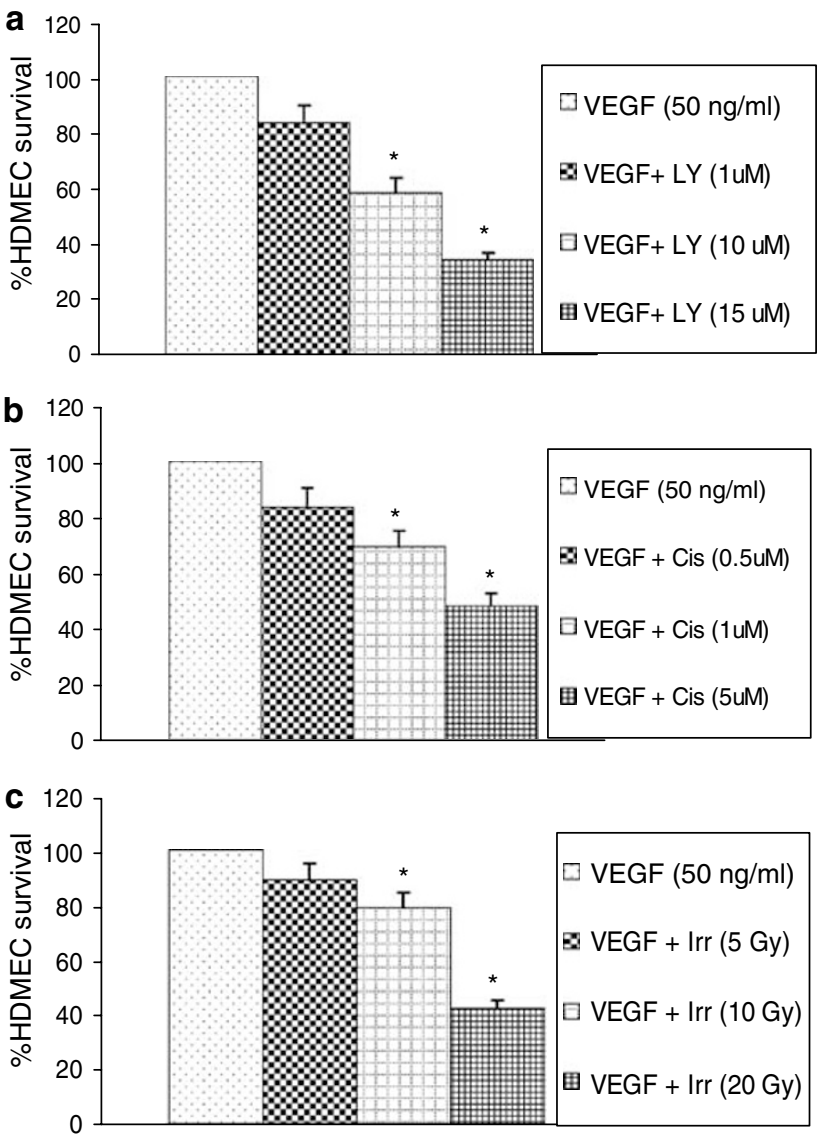

Figure 1 PI3 K inhibitor (LY294002), cisplatin and $\gamma$-irradiation induce dose-dependent increase in EC death. HDMECs were cultured in $60 \mathrm{~mm}$ dishes containing a thin layer of type 1 collagen gel for $72 \mathrm{~h}$ in the presence of VEGF $(50 \mathrm{ng} / \mathrm{ml})$. (a) HDMECs were treated with 1,10 and $15 \mu \mathrm{M}$ doses of LY294002 (LY). (b) HDMECs were treated with $0.5,1$ and $5 \mu \mathrm{M}$ doses of cisplatin (Cis). (c) HDMECs were treated with 5, 10 and $15 \mathrm{~Gy}$ doses of $\gamma$-irradiator (Irr). The cells were further incubated at $37^{\circ} \mathrm{C}$ for 3 days. On days 0 and 3 , five random high-power fields $(\times 200)$ of each sample group from three independent experiments were photographed and counted using Metamorph software. The percentage of HDMEC survival was calculated for each group by dividing day 3 counts by day 0 counts and multiplying by 100. *, Represents a significant difference $(P<0.05)$ as compared to the control group.

showed remarkably higher inhibition of endothelial survival (58\%).

OSCC-3 cells also exhibited a dose-dependent decrease in survival in response to LY294002, cisplatin and $\gamma$-irradiation treatment. PI3 $\mathrm{K}$ inhibitor (LY294002) induced a 28 and $46 \%$ inhibition of OSCC-3 survival at 10 and $15 \mu \mathrm{M}$ doses, respectively. This was significantly lower than the inhibition of HDMEC survival ( 42 and $64 \%$ ) at the same doses (Figure 2a). In contrast, $\gamma$-irradiation treatment was considerably more effective in inhibiting OSCC3 survival $(21,42$ and $71 \%$ at 5,10 and $15 \mathrm{~Gy}$, respectively) as compared to HDMEC (11, 21 and $58 \%$ ) at the same doses (Figure 2b). Cisplatin treatment showed similar effectiveness in both OSCC-3 and HDMEC (Figures 1b and 2b).
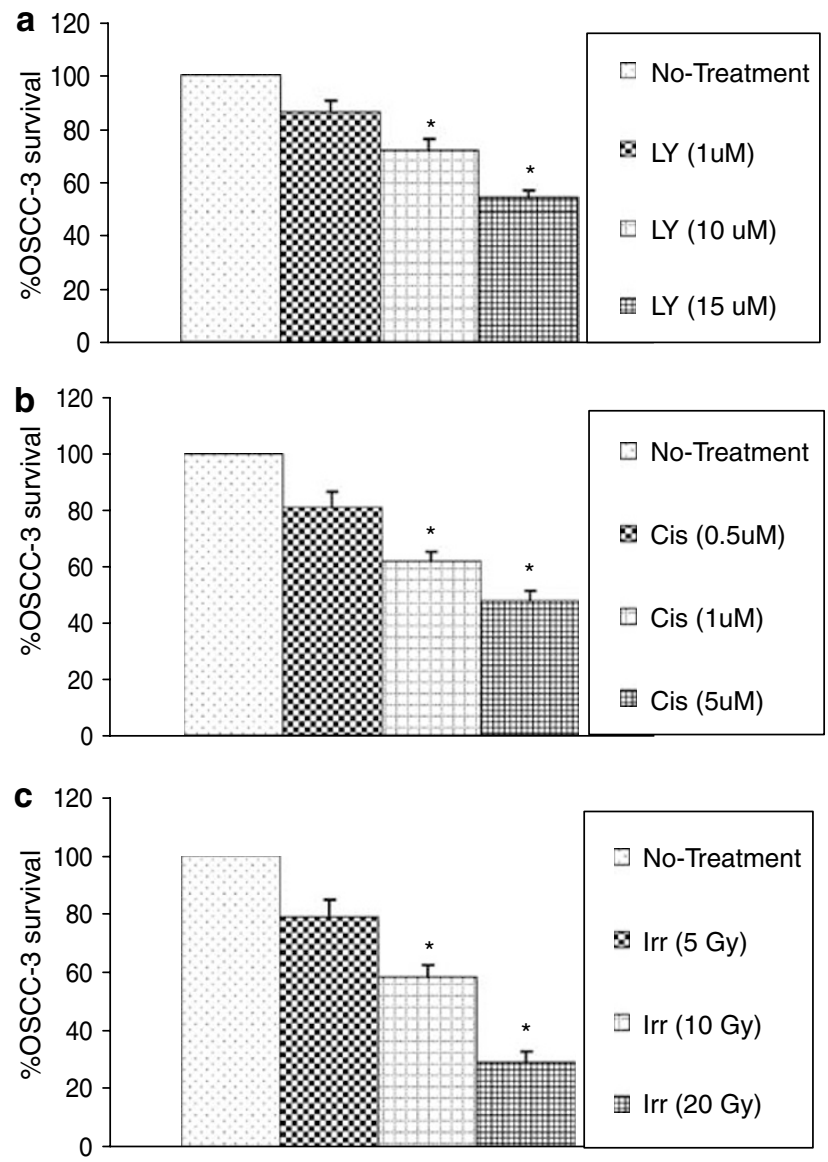

Figure 2 PI3 K inhibitor (LY294002), cisplatin and $\gamma$-irradiation induce dose-dependent increase in tumor cell death. OSCC-3 cells were cultured in $60 \mathrm{~mm}$ dishes. (a) OSCC-3 cells were treated with 1, 10 and $15 \mu \mathrm{M}$ doses of LY294002 (LY). (b) OSCC-3 cells were treated with $0.5,1$ and $5 \mu \mathrm{M}$ doses of cisplatin (Cis). (c) OSCC-3 cells were treated with 5, 10 and 15Gy doses of $\gamma$ irradiator (Irr). The cells were further incubated at $37^{\circ} \mathrm{C}$ for 3 days. On days 0 and 3 , five random high-power fields $(\times 200)$ of each sample group from three independent experiments were photographed and counted using Metamorph software. The percentage of OSCC-3 survival was calculated for each group by dividing day 3 counts by day 0 counts and multiplying by $100 .{ }^{*}$, Represents a significant difference $(P<0.05)$ as compared to the control group.

\section{Combination Treatment with LY294002, Cisplatin, and Irradiation Showed Significant Decrease in HDMEC and OSCC-3 Survival}

HDMECs were cultured in $60 \mathrm{~mm}$ dishes on a thin layer of collagen and cultured for $72 \mathrm{~h}$ at $37^{\circ} \mathrm{C}$ in the presence of VEGF and then treated with LY294002, cisplatin, and $\gamma$-irradiation either alone or in combination and cultured for an additional $72 \mathrm{~h}$. For combination treatment studies, we selected doses of LY294002 $(1 \mu \mathrm{M})$, cisplatin $(0.5 \mu \mathrm{M})$ and $\gamma$-irradiation (10Gy) that did not markedly reduce HDMEC survival by themselves. LY294002 $(1 \mu \mathrm{M})+$ cisplatin $(0.5 \mu \mathrm{M})$, LY294002 $(1 \mu \mathrm{M})+$ irradiation (10 Gy), and cisplatin $(0.5 \mu \mathrm{M})+$ irradiation (10 Gy) combination treatments showed 39, 41 and $36 \%$ inhibition of HDMEC survival, respectively 

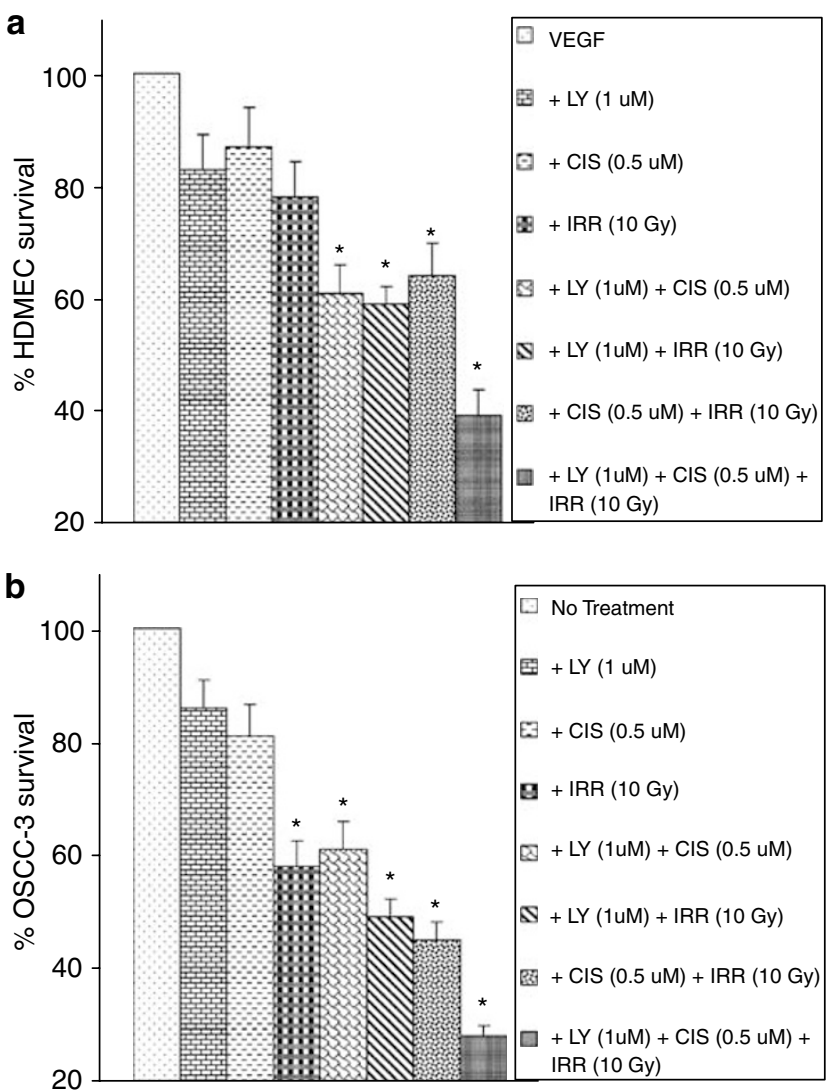

Figure 3 Combination treatment with LY294002, cisplatin and irradiation showed significantly higher inhibition of HDMEC and OSCC-3 survival. (a) HDMECs were cultured in $60 \mathrm{~mm}$ dishes containing a thin layer of type 1 collagen gel for $72 \mathrm{~h}$ in the presence of VEGF $(50 \mathrm{ng} / \mathrm{ml})$. (b) OSCC-3 cells were plated in $60 \mathrm{~mm}$ dishes and incubated overnight at $37^{\circ} \mathrm{C}$. HDMECs and OSCC-3 cells were then treated with LY294002 (LY, $1 \mu \mathrm{M}$ ), cisplatin (Cis, $0.5 \mu \mathrm{M}), \gamma$-irradiation (Irr, 10 Gy), LY $(1 \mu \mathrm{M})+$ Cis $(0.5 \mu \mathrm{M})$, LY $(1 \mu \mathrm{M})+\operatorname{Irr}(10 \mathrm{~Gy})$, Cis $(0.5 \mu \mathrm{M})+\operatorname{Irr}(10 \mathrm{~Gy})$ or LY $(1 \mu \mathrm{M})+$ Cis $(0.5 \mu \mathrm{M})+\operatorname{Irr}(10 \mathrm{~Gy})$. The cells were further incubated at $37^{\circ} \mathrm{C}$ for 3 days. On days 0 and 3 , five random high-power fields $(\times 200)$ of each sample group from three independent experiments were photographed and counted using Metamorph software. The percentage of HDMEC and OSCC-3 survival was calculated for each group by dividing day 3 counts by day 0 counts and multiplying by $100 .{ }^{*}$, Represents a significant difference $(P<0.05)$ as compared to the control group.

(Figure 3a). Combination treatment with LY294002 $(1 \mu \mathrm{M})+$ cisplatin $(0.5 \mu \mathrm{M})+$ irradiation (10 Gy) together resulted in significantly higher inhibition of HDMEC survival (61\%) as compared to treatment with a single agent or in combination of two agents (Figure 3a).

Next, we analyzed the effect of combination treatment on OSCC-3 survival. OSCC-3 cells were cultured in $60 \mathrm{~mm}$ dishes and treated with LY294002, cisplatin or irradiation either alone or in combination and incubated at $37^{\circ} \mathrm{C}$ for $72 \mathrm{~h}$. Similar to HDMEC, OSCC-3 treated with a double combination treatment of LY294002 $(1 \mu \mathrm{M})+$ cisplatin $(0.5 \mu \mathrm{M})$, LY294002 $(1 \mu \mathrm{M})+$ irradiation (10 Gy) and cisplatin $(0.5 \mu \mathrm{M})+$ irradiation (10 Gy) showed significantly higher inhibition of OSCC-3 survival (Figure 3b). Cisplatin and irradiation combination was most effective in inhibiting OSCC-3 survival, whereas LY294002 and cisplatin was least effective in inhibiting OSCC-3 survival. Combination treatment with LY294002, cisplatin, and irradiation together showed a significant additive effect in inhibiting OSCC-3 survival (72\%).

\section{Combination Treatment Significantly Inhibits VEGF-Mediated EC Tube Formation In Vitro}

We evaluated the effect of combination treatment on angiogenesis by performing an endothelial tube formation assay, in vitro. VEGF stimulated ECs were treated with LY294002, cisplatin or irradiation either alone or in combination and cultured on growth factor reduced Matrigel for $16-18 \mathrm{~h}$ at $37^{\circ} \mathrm{C}$. VEGF stimulated HDMEC showed significantly greater tube formation as compared to nonstimulated controls (Figure 4a and b). LY294002 $(1 \mu \mathrm{M})$, cisplatin $(0.5 \mu \mathrm{M})$ and irradiation (10 Gy) showed 8 , 8 and 18\% inhibition of HDMEC tube formation, respectively. ECs treated with a combination of LY294002 $(1 \mu \mathrm{M})+$ cisplatin $(0.5 \mu \mathrm{M}) ; \quad$ LY294002 $(1 \mu \mathrm{M})+$ irradiation $(10 \mathrm{~Gy})$ or cisplatin $(0.5 \mu \mathrm{M})+$ irradiation (10 Gy) showed significantly higher inhibition of tube formation (35, 42 and $43 \%$, respectively). Similar to the HDMEC survival assay, combination treatment with LY294002, cisplatin, and irradiation together showed significantly higher inhibition of VEGF-mediated HDMEC tube formation $(75 \%)$ as compared to each of these agents used alone (Figure 4a and b).

\section{Combination Treatment Significantly Inhibits Tumor Growth In Vivo}

The effects of combination treatment on tumor growth in vivo was examined using our SCID mouse model for tumor growth and tumor angiogenesis. ${ }^{25,26}$ We performed two sets of experiments for tumor growth studies. In the first set of experiments, we investigated the effect of a combination therapy on tumor growth and tumor angiogenesis as well as on normal angiogenesis in the same animals at a distal site. In this study, the animals were killed at day 14 and tumors were carefully retrieved, measured with calipers, and weighed on an electronic balance. We had selected day 14 for these experiments because we had earlier observed that HDMECs in SCID mouse model show peak tumor angiogenesis between 7 and 14 days. ${ }^{25,26}$ Animals treated with LY294002 $(5 \mathrm{mg} / \mathrm{kg})$, cisplatin $(5 \mathrm{mg} / \mathrm{kg})$ or irradiation (10 Gy) alone showed 28,27 , and $32 \%$ decrease in tumor size, respectively (Figure 5). Combination treatment with two agents together more than doubled the inhibition of tumor growth $(72-80 \%)$ as compared to each of these agents given alone. Combination treatment with LY294002, cisplatin 


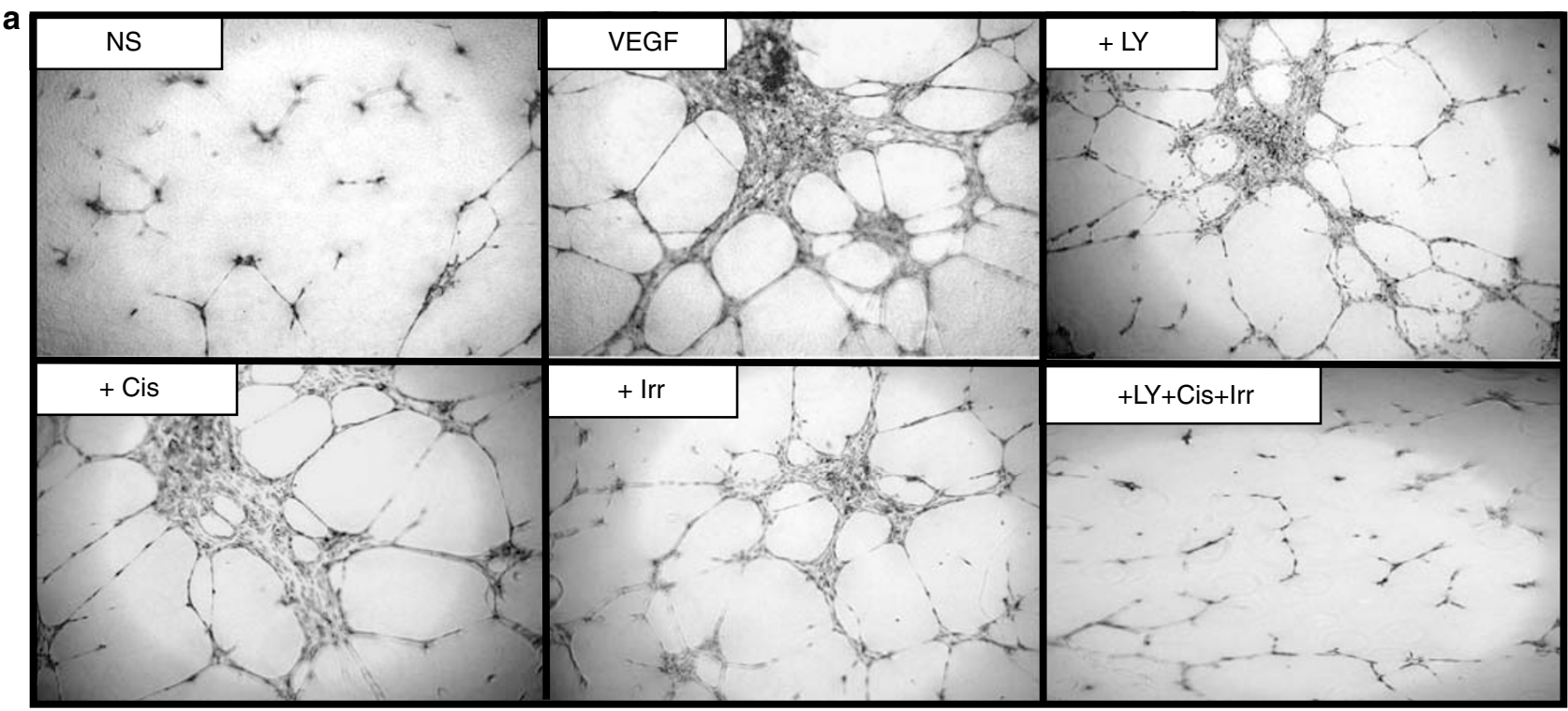

b

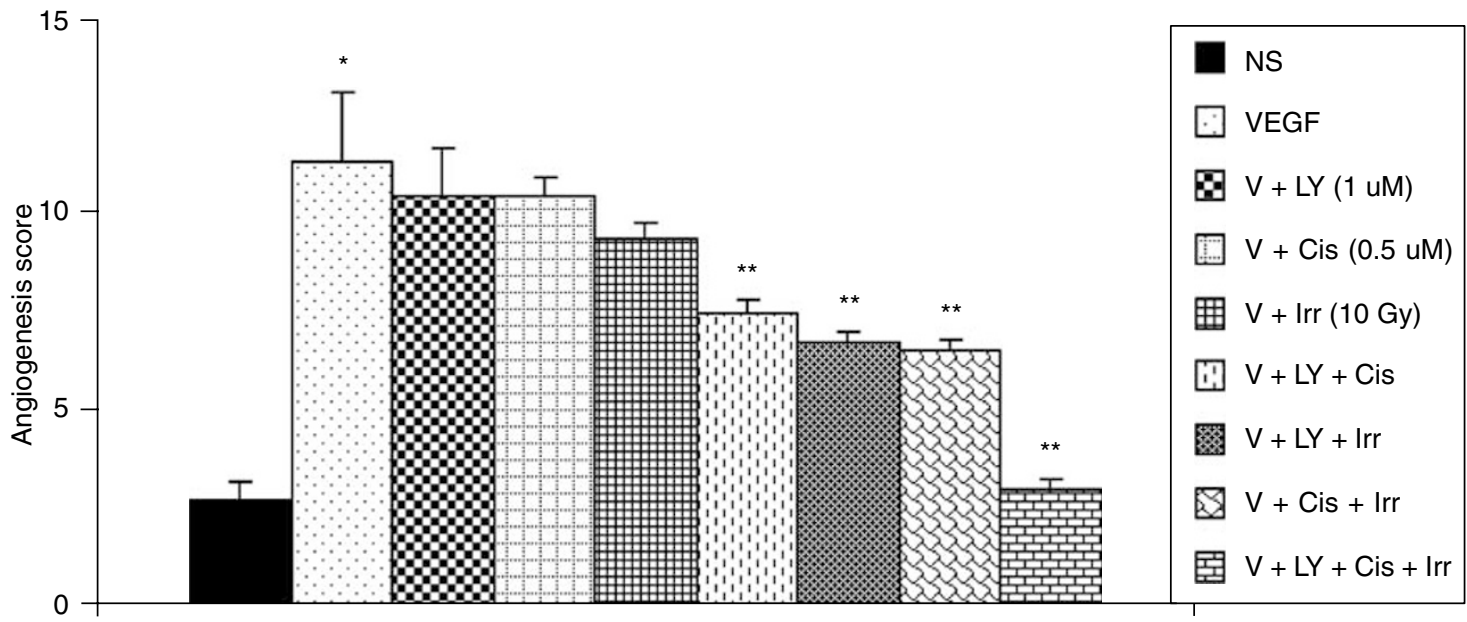

Figure 4 Combination treatment significantly inhibits VEGF-mediated EC tube formation in vitro. The Matrigel in vitro HDMEC tube formation assay was performed in eight-well chamber slides. (a) Photomicrographs of representative assays for no-treatment (NT), VEGF (V), VEGF + LY294002 (V + LY), VEGF + cisplatin (V + Cis), VEGF $+\gamma$-irradiation (V + Irr) and VEGF + LY294002 + cisplatin $+\gamma$ irradiation (V+LY + Cis + Irr). (b) Quantitative data for EC tube formation expressed as angiogenic score \pm s.e. from three independent experiments. ${ }^{*}$ and ${ }^{* *}$, represents a significant increase and significant decrease $(P<0.05)$, respectively, between the test and respective control group.

and irradiation together resulted in more than $90 \%$ reduction in tumor size, which was significantly lower than each of the treatment group alone or the two agent combination groups.

In the second set of experiments, we investigated the effect of combination therapy on tumor growth using two cell lines OSCC-3 and LnCap. The tumor growth was assessed twice a week by quantifying the tumor volume as described in Materials and methods. As observed earlier, treatment with LY294002 $(5 \mathrm{mg} / \mathrm{kg})$, cisplatin $(5 \mathrm{mg} / \mathrm{kg})$ or irradiation (10 Gy) alone did not significantly inhibit growth of both OSCC-3 (Figure 6a) and LnCap (6b) tumors. In contrast, combination treatment with LY294002, cisplatin and irradiation significantly inhibited both oral squamous carcinoma (OSCC-3,
Figure 6a) as well as prostate cancer (LnCap, Figure $6 \mathrm{~b}$ ) growth. These results suggest that this combination therapy consisting of low doses of PI3 K inhibitor (LY294002), chemotherapeutic agent (cisplatin) and irradiation may be an effective treatment strategy for oral squamous carcinomas as well as prostate cancer.

We next analyzed the effect of combination treatment on tumor cell apoptosis. Paraffinembedded tissue sections were stained with in situ TUNEL assay kits as well as with activated caspase-3 antibody. Very few TUNEL-positive cells $(4 \%)$ were observed in the no-treatment control group (Figure $7 \mathrm{a}$ and b). Tumor samples from animals treated with LY294002, cisplatin or irradiation alone showed 18, 16 and 20\% TUNEL- 


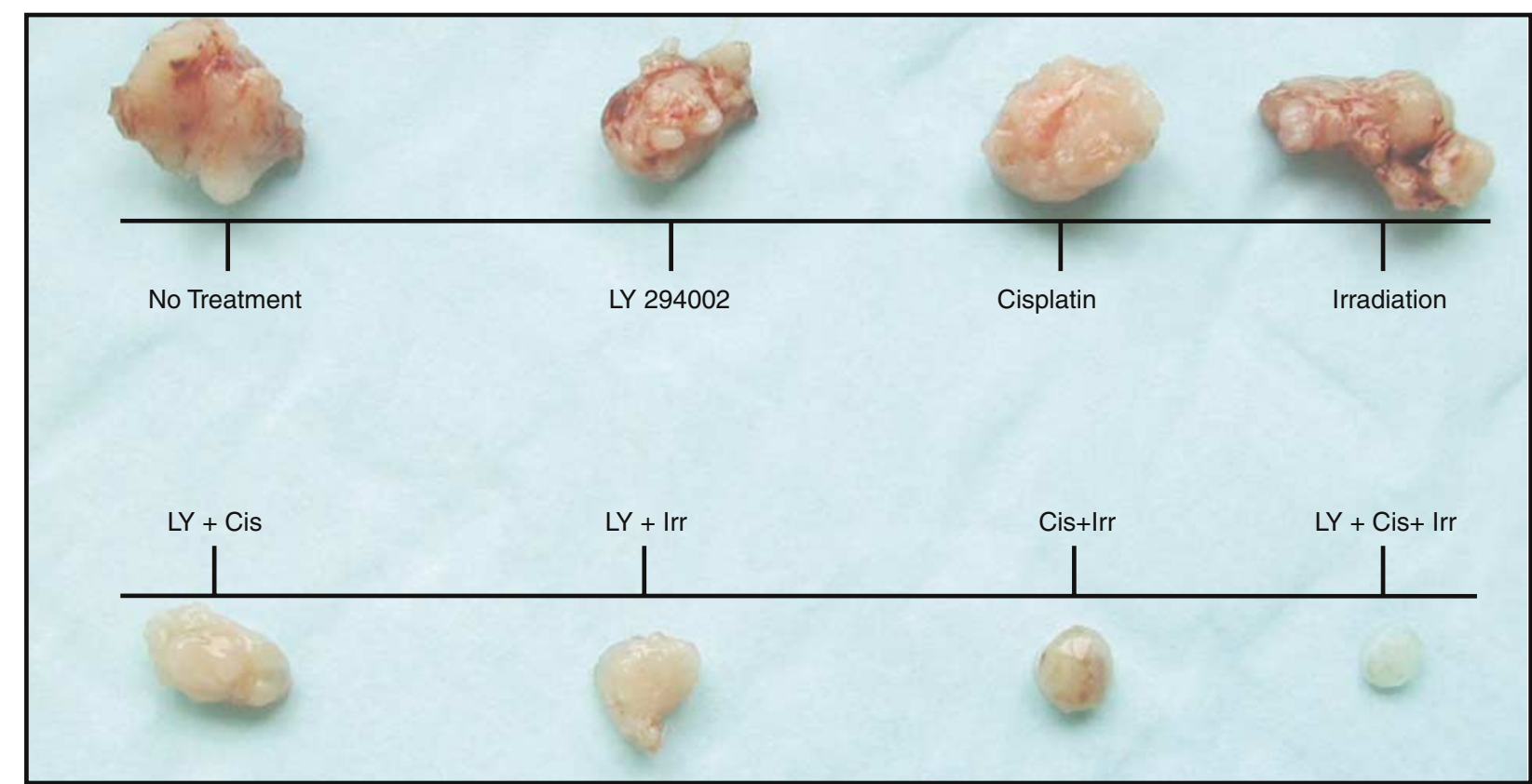

b
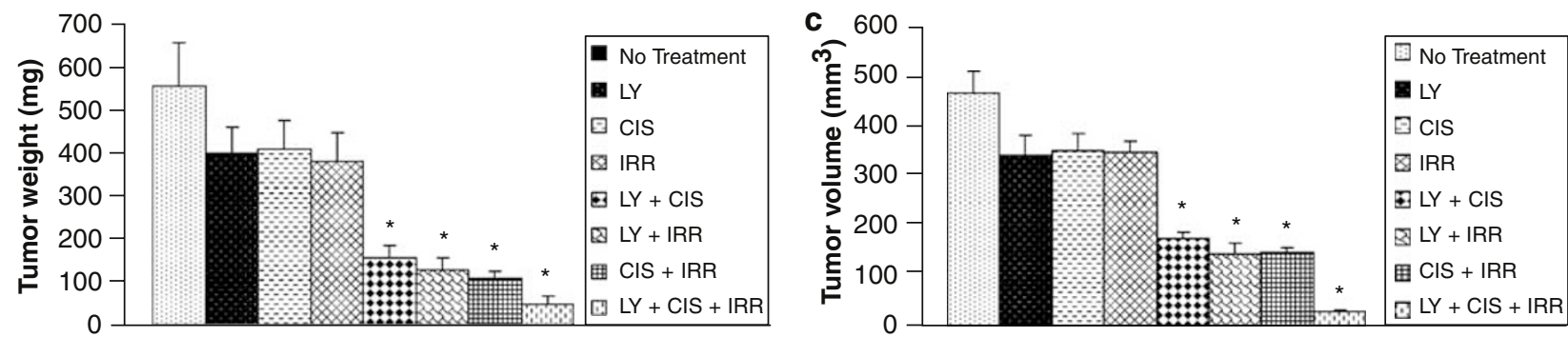

Figure 5 Combination treatment significantly inhibits tumor growth in vivo. In all, $9 \times 10^{5}$ HDMEC and $1 \times 10^{5}$ OSCC-3 cells were mixed with $100 \mu \mathrm{l}$ of Matrigel and injected into the rear hind limb of SCID mice (10 mice/group). On days 2, 5 and 10 mice were treated with LY294002 $(5 \mathrm{mg} / \mathrm{kg})$ and/or cisplatin $(5 \mathrm{mg} / \mathrm{kg})$ via I.P. injections. On day 3 the mice's rear hind limb, where the tumor and ECs were implanted, were exposed to irradiation ( $10 \mathrm{~Gy}$ ). After 14 days, the animals were killed; tumors were carefully retrieved, measured with calipers, and weighed on an electronic balance. (a) Representative tumor tissues from no-treatment, LY294002 (LY), cisplatin (Cis), irradiation (Irr), LY + Cis, LY + Irr, Cis + Irr and LY + Cis + Irr. (b) The results shown are the mean tumor weight + s.e.; ${ }^{*} P<0.05$. (c) The results shown are the mean tumor volume + s.e.; ${ }^{*} P<0.05$

positive cells, respectively. Tumor samples from combination treatment with LY294002 + cisplatin; LY294002 + irradiation or cisplatin + irradiation showed 39, 42, and 41\% TUNEL-positive cells, respectively. Significantly, higher TUNEL-positive cells $(92 \%)$ were observed in tumor samples from animals treated with LY294002, cisplatin and irradiation together (Figure 7a and b). Similarly, tumor samples from animals treated with LY294002, cisplatin and irradiation showed significantly higher activated caspase-3-positive cells (56\%) as compared to the no-treatment group $(5 \%)$ or animals treated with LY294002, cisplatin and irradiation separately (19, 15 and 21\%, respectively).

\section{Combination Treatment Preferentially Target Tumor Angiogenesis}

The effect of combination treatment on tumor angiogenesis as well as physiological angiogenesis was also studied. For tumor angiogenesis studies, OSCC-3 and HDMECs were mixed with growth factor-reduced Matrigel and implanted into the rear upper limb of SCID mice. In the same animals, VEGF-incorporated PLG-scaffolds seeded with HDMECs were implanted subcutaneously in the dorsal region. We first did a dose response study to determine the appropriate amount of VEGF incorporation in PLG-scaffolds for normal angiogenesis. The $0.25 \mu \mathrm{g}$ VEGF dose was optimal in inducing significant angiogenesis and this dose was used for all the subsequent studies. Human blood vessel formation in these tumors as well as scaffolds was analyzed by immunostaining using anti-human Von Willebrand factor and anti-human CD31 antibodies. Animals treated with LY294002, cisplatin or irradiation alone did not show a significant decrease in tumor vessel area (Figure 8a and b). Combination treatment with LY294002 + cisplatin; LY294002+ irradiation or cisplatin + irradiation showed a significant decrease in tumor vessel area $(45,51$, and 

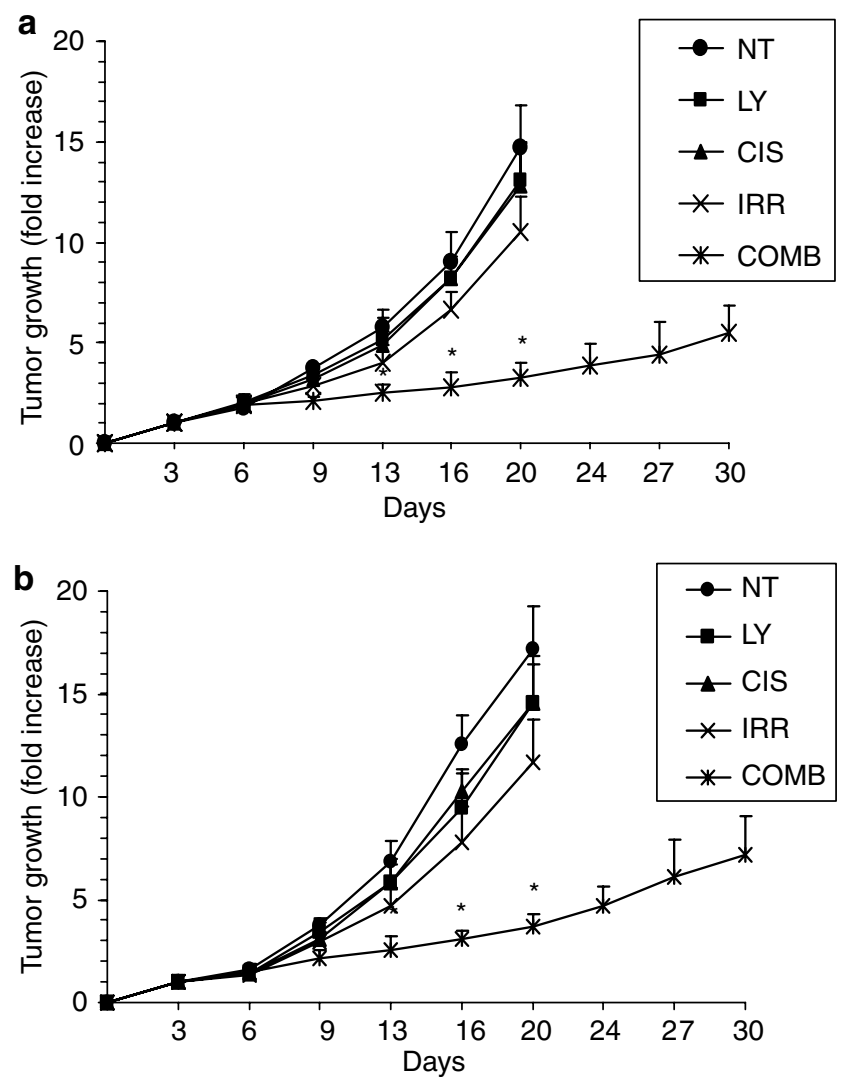

Figure 6 Combination treatment significantly inhibited OSCC-3 and LnCap tumor growth in vivo. In all, $9 \times 10^{5}$ HDMEC and $1 \times 10^{5}$ OSCC-3 or LnCap cells were mixed with $100 \mu \mathrm{l}$ of Matrigel and injected into both rear hind limbs of SCID mice (five mice/ group). On days 6, 9 and 14 the mice were treated with LY294002 $(5 \mathrm{mg} / \mathrm{kg})$ and/or cisplatin $(5 \mathrm{mg} / \mathrm{kg})$ via I.P. injections. On day 7 mice's rear hind limb, where the tumor and ECs were implanted, were exposed to irradiation (10 Gy). Tumor volume measurements were taken, as described in Materials and methods, starting from day 1 and continued twice a week till day 30. Data were calculated at the percentage of tumor volume at day 3 and graphed as fold change in tumor volume + s.e. for each treatment group. (a) Tumor progression curve for OSCC-3 for no-treatment (NT), LY294002 (LY), cisplatin (CIS), irradiation (Irr) and LY + CIS + IRR groups, ${ }^{*} P<0.05$. (b) Tumor progression curve for LnCap for no-treatment (NT), LY294002 (LY), cisplatin (CIS), irradiation (Irr) and LY + CIS + IRR groups, ${ }^{*} P<0.05$.

$44 \%$, respectively). Combination treatment with LY294002, cisplatin and irradiation together was most effective by inhibiting more than $90 \%$ of tumor angiogenesis (Figure 8a and b). In contrast to tumor angiogenesis, combination treatment with LY294002, cisplatin and irradiation showed only $29 \%$ decrease in normal angiogenesis as assessed by vessel area in scaffold sections (Figure 9a and b). We have shown data in Figures 8 and 9 obtained from tumors and scaffold sections stained with anti-human Von Willebrand factor. Similar results were observed in tumors and scaffold sections stained with anti-human CD31 antibody (data not shown).

\section{Discussion}

Recent advances in the development of targetspecific pharmacological signaling inhibitors have provided hope for enhancing the efficacy of traditional therapies including radiation therapy. ${ }^{27} \mathrm{~A}$ major challenge for developing combination treatments is the identification of specific target molecule(s) that can provide the highest degree of synergistic antitumor activity with traditional therapies, in vivo. It is therefore important to address the possible mechanisms by which tumor cells and endothelial cells lining the tumors vessels acquire resistance. We have previously shown that activation of the PI3K/Akt survival pathway by VEGF protects ECs from the apoptotic effects of $\gamma$-irradiation. ${ }^{19}$ The PI3K/Akt pathway is also used by other growth factors like basic fibroblast growth factor and platelet-derived growth factor to mediate angiogenesis and EC protection. ${ }^{28}$ We therefore hypothesize that treatment with PI3 K inhibitor prior to irradiation may be a useful strategy to overcome resistance in the ECs lining the tumor blood vessels and thereby enhance the effectiveness of $\gamma$-radiation and chemotherapy.

To test this hypothesis, we have performed combination treatment studies in vitro as well as in vivo with PI3 K inhibitor (LY294002), cisplatin and ionizing radiation. For in vitro EC survival assays, we cultured endothelial cells in the presence of VEGF for 3 days before exposing them to LY294002, cisplatin or irradiation. This was performed to replicate the conditions similar to the in vivo tumor environment where ECs are constantly exposed to high levels of growth factors including VEGF. We first performed a dose-response study with PI3 K inhibitor (LY294002), cisplatin, and ionizing radiation on ECs (HDMECs) as well as tumor cells (OSCC-3). LY294002 showed a significantly higher level of inhibition of HDMEC survival as compared to OSCC- 3 cells at the same doses. A number of studies have shown that enhanced expression of epidermal growth factor receptor (EGFR) correlate positively with the acquisition of resistance by squamous cell carcinoma. ${ }^{29}$ EGFR mediates resistance in squamous cell carcinoma by activating a number of effectors including RasMAPK and PI3 K. ${ }^{30}$ Therefore, it is possible that OSCC-3 cells may be using multiple signaling pathways for survival downstream of EGFR. Where as ECs predominantly use the PI3K/Akt pathway for survival. ${ }^{19}$ In contrast to LY294002, $\gamma$-irradiation showed significantly higher inhibition of OSCC-3 survival as compared to ECs at the same doses. ECs have been shown to be quite resistant to radiation therapy and require relatively high doses of radiation to induce significant endothelial cell death. ${ }^{18,19}$ Conversely, higher radiosensitivity of OSCC-3 cells could be due to their rapid cell division, making the chromatin susceptible to higher DNA damage against ionizing radiation. Cisplatin was equally 


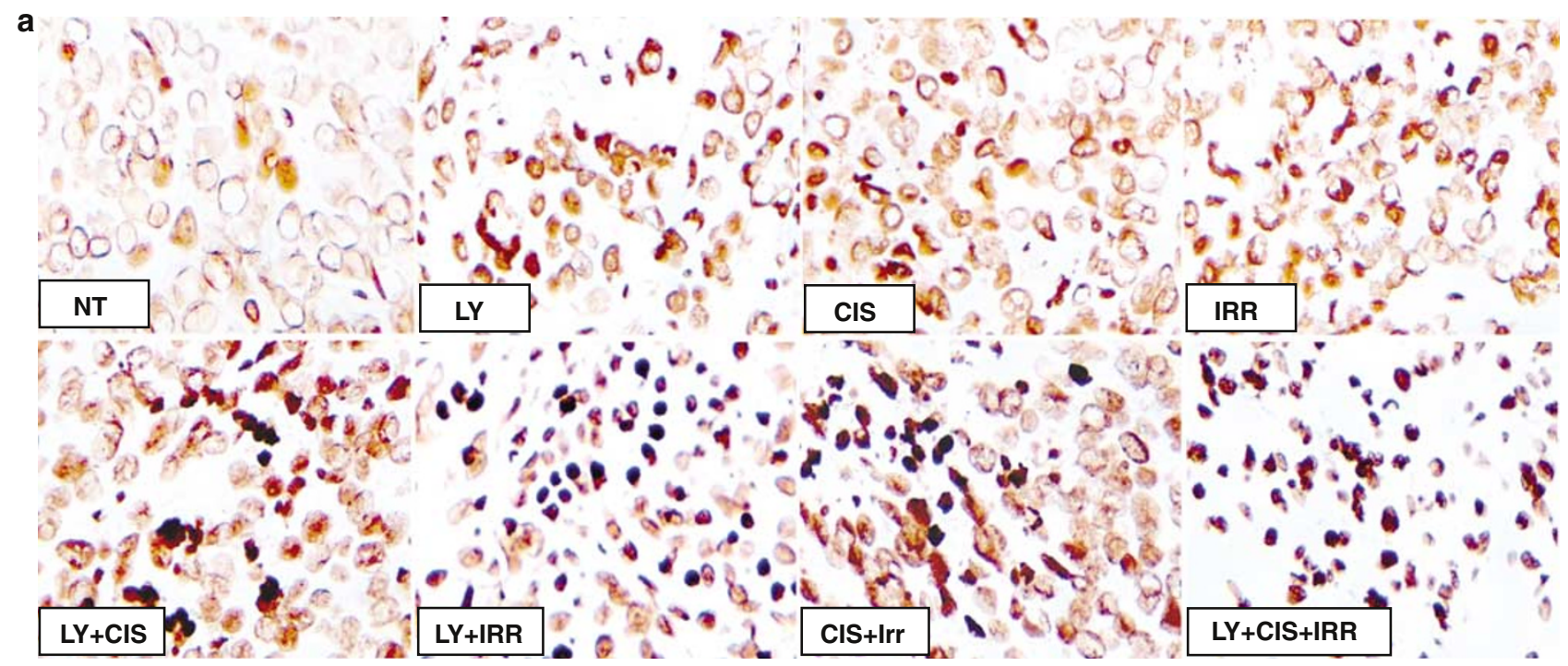

b

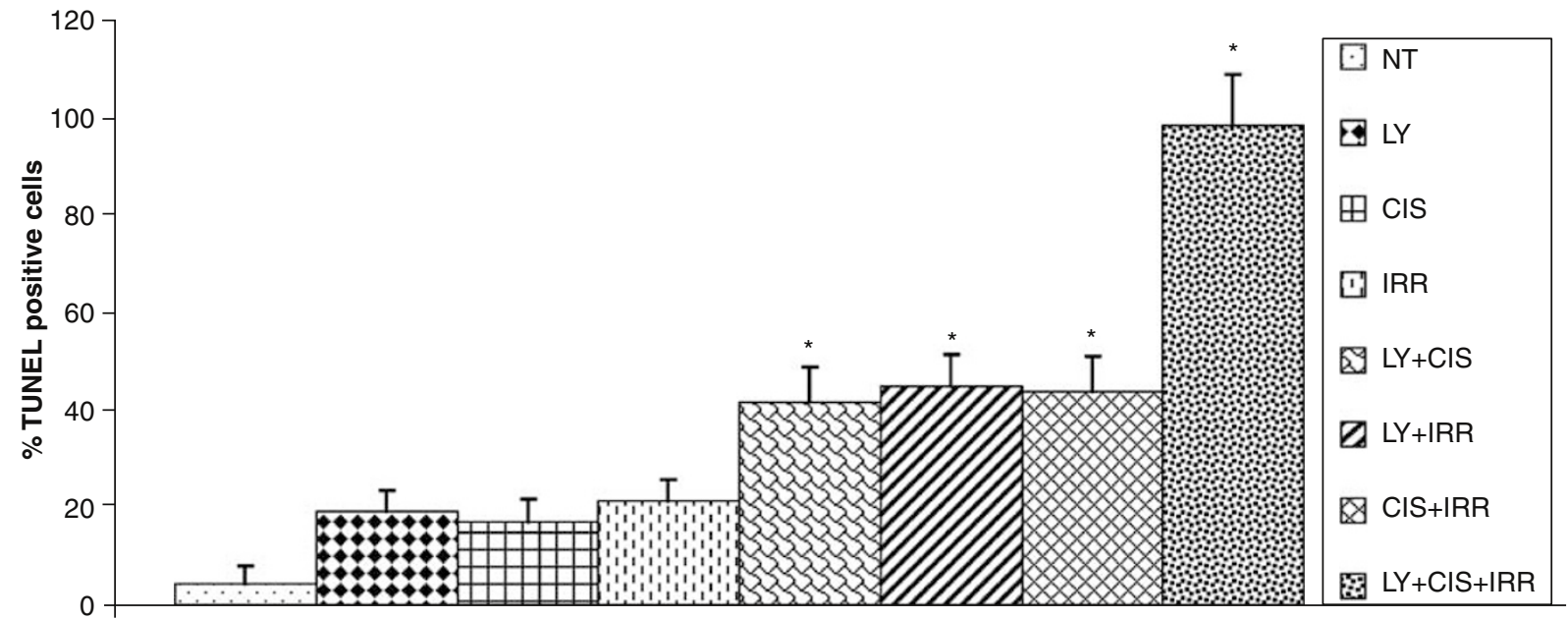

Figure 7 Tumor samples from animals treated with combination treatment showed significantly higher apoptosis. Paraffin-embedded tumor sections were stained for TUNEL-positive cells using KLENOW-FragEL DNA Fragment Detection Kit. (a) Representative photomicrographs of treatment groups; no-treatment (NT), LY294002 (LY), cisplatin (Cis), irradiation (Irr), LY + Cis, LY + Irr, Cis + Irr and $\mathrm{LY}+\mathrm{Cis}+$ Irr. (b) The results are shown as \% TUNEL-positive cells + s.e. *, Represent a significant increase $(P<0.05)$ in TUNEL-positive cells as compared to the control.

effective in mediating HDMEC and OSCC-3 cell death.

In the combination treatment studies, we selected doses of LY294002 $(1 \mu \mathrm{M})$, cisplatin $(0.5 \mu \mathrm{M})$, and $\gamma$ irradiation (10 Gy) that by themselves did not show significant inhibition of HDMEC survival. The rationale for selecting these low doses was to have the greatest possible synergistic antitumor effect without the serious side effects that are often associated with the maximum tolerated doses of radiation and chemotherapy. Combination treatment with LY294002 $(1 \mu \mathrm{M})$, cisplatin, $(0.5 \mu \mathrm{M})$ and $\gamma$ irradiation (10 Gy) significantly decreased EC and OSCC-3 survival as well as significantly decreasing EC tube formation, in vitro. To determine if the observed in vitro synergy between LY294002, cisplatin and ionizing radiation extends to the in vivo setting, we used our SCID mouse model to study the effect of combination treatment on tumor growth and tumor angiogenesis as well as normal angiogenesis in two sets of experiments. In the first set of experiments, we killed animals at day 14 and carefully retrieved tumors and PLG scaffolds. The rationale for choosing day 14 was based on our earlier findings that HDMECs in our SCID mouse model show peak tumor angiogenesis as well as normal angiogenesis between 7 and 14 days. ${ }^{25,26}$ In the second set of experiments, we investigated the effect of combination therapy on tumor growth over a 30-day period by quantifying tumor volumes twice a week. Combination treatment with low doses of PI3 K inhibitor (LY294002), cisplatin and irradiation was very effective and showed a significant decrease in tumor size as compared to the no-treatment group or single-treatment group alone. A number of studies have shown that PI3 K inhibitor (LY294002) 
a

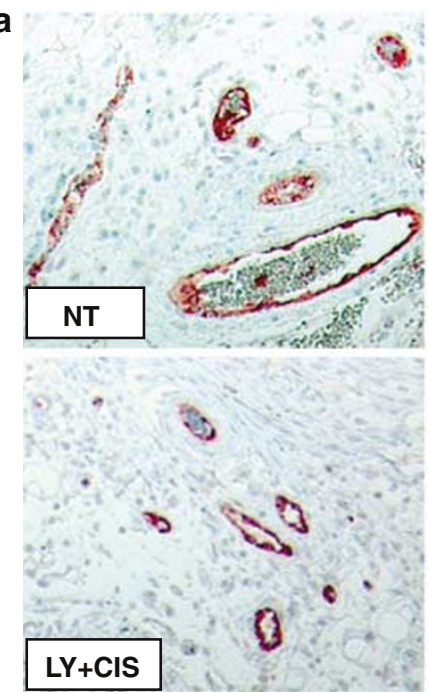

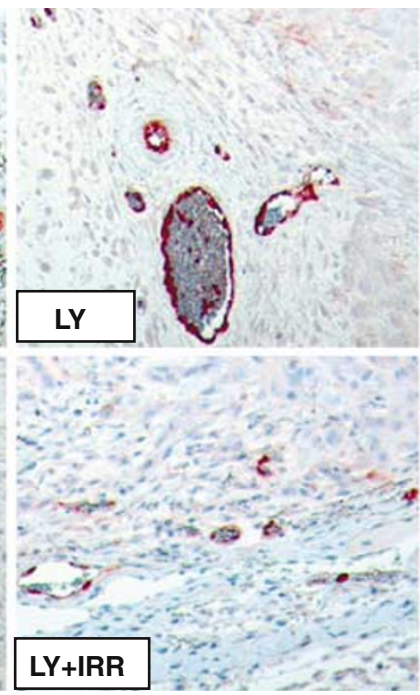
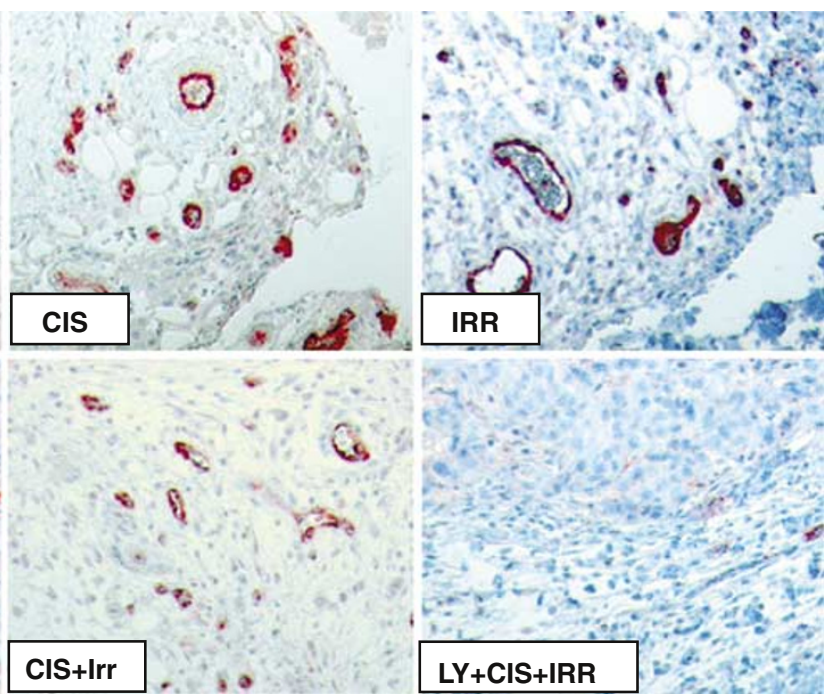

b

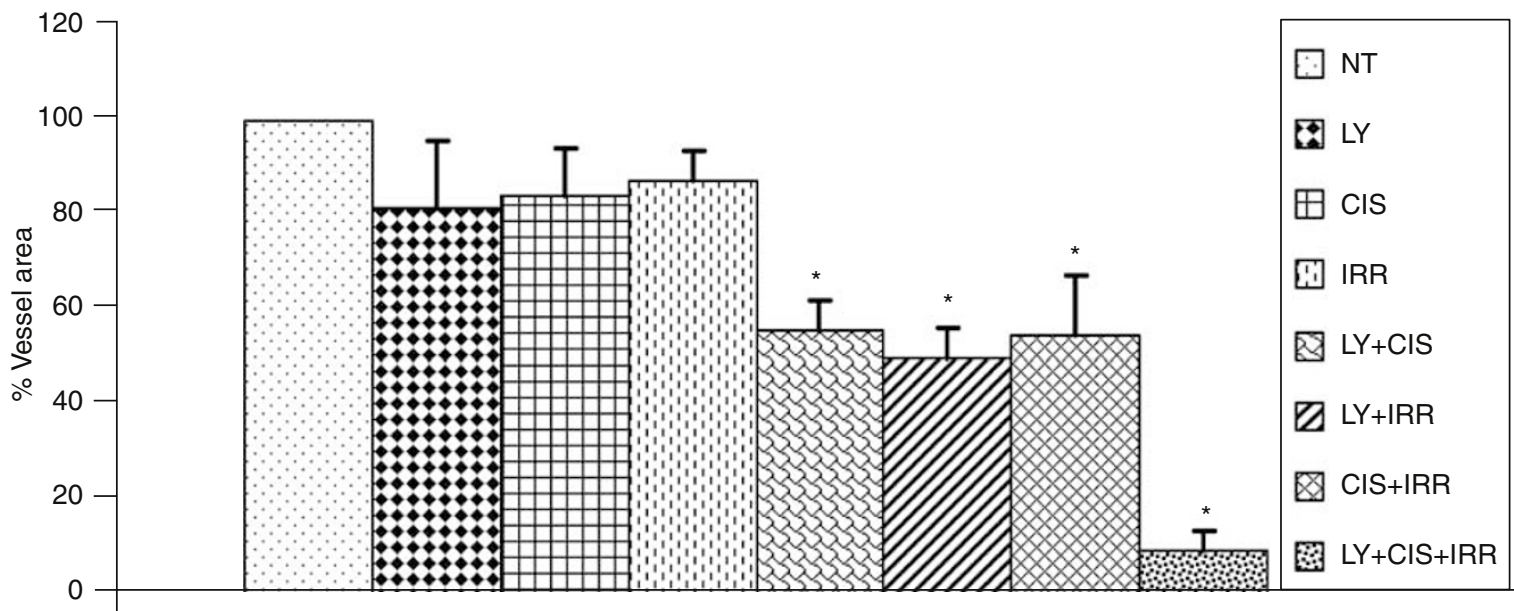

Figure 8 Combination treatment significantly inhibited tumor angiogenesis. Paraffin-embedded tumor sections were stained for tumor blood vessels using anti-human Von Willebrand factor antibodies. (a) Representative photomicrographs of treatment groups; notreatment (NT), LY294002 (LY), cisplatin (Cis), irradiation (Irr), LY + Cis, LY + Irr, Cis + Irr and LY + Cis + Irr. (b) Vessel area in the tumor samples was quantified using Metamorph software and the results are expressed as \% vessel area + s.e. *, Represent a significant decrease $(P<0.05)$ in \% vessel area as compared to the control.

can significantly enhance the radiosensitivities of tumors. ${ }^{31-33}$ However, some of these studies have used very high doses of PI3 K inhibitor. ${ }^{31}$ In this study, we have combined low doses of PI3 K inhibitor (LY294002) and cisplatin along with ionizing radiation to significantly inhibit tumor growth. This inhibition of tumor growth by combination therapy could be due to its effect on tumor cell clonogens ${ }^{16}$ as well as inhibition of tumor angiogenesis. This combination treatment did not cause any animal mortality nor did it induce any major systemic toxicity such as dry scaly skin or respiratory depression, which has been reported in animals treated with high doses $(50-100 \mathrm{mg} / \mathrm{kg})$ of LY294002. ${ }^{31}$

The combination treatment with LY294002, cisplatin and irradiation also significantly decreased tumor angiogenesis. In contrast, the combination treatment in the same animals showed significantly lower inhibition of physiological angiogenesis. It is also possible that rapid tumor blood vessel growth, under the influence of growth factors secreted by tumor cells, make them much more sensitive to the combination of PI3 K inhibitor (LY294002) and DNA damaging agents (ionizing radiation and cisplatin). These results suggest that this combination therapy treatment, which preferentially targets tumor angiogenesis may also be effective in the treatment of other solid tumors. To investigate this, we used a human prostate cancer cell line (LnCap) and studied the effect of combination therapy on tumor progression. Results were similar to what we observed with combination treatment of human oral squamous carcinoma.

In conclusion, we have demonstrated in this study that the combination of low doses of PI3 K inhibitor 


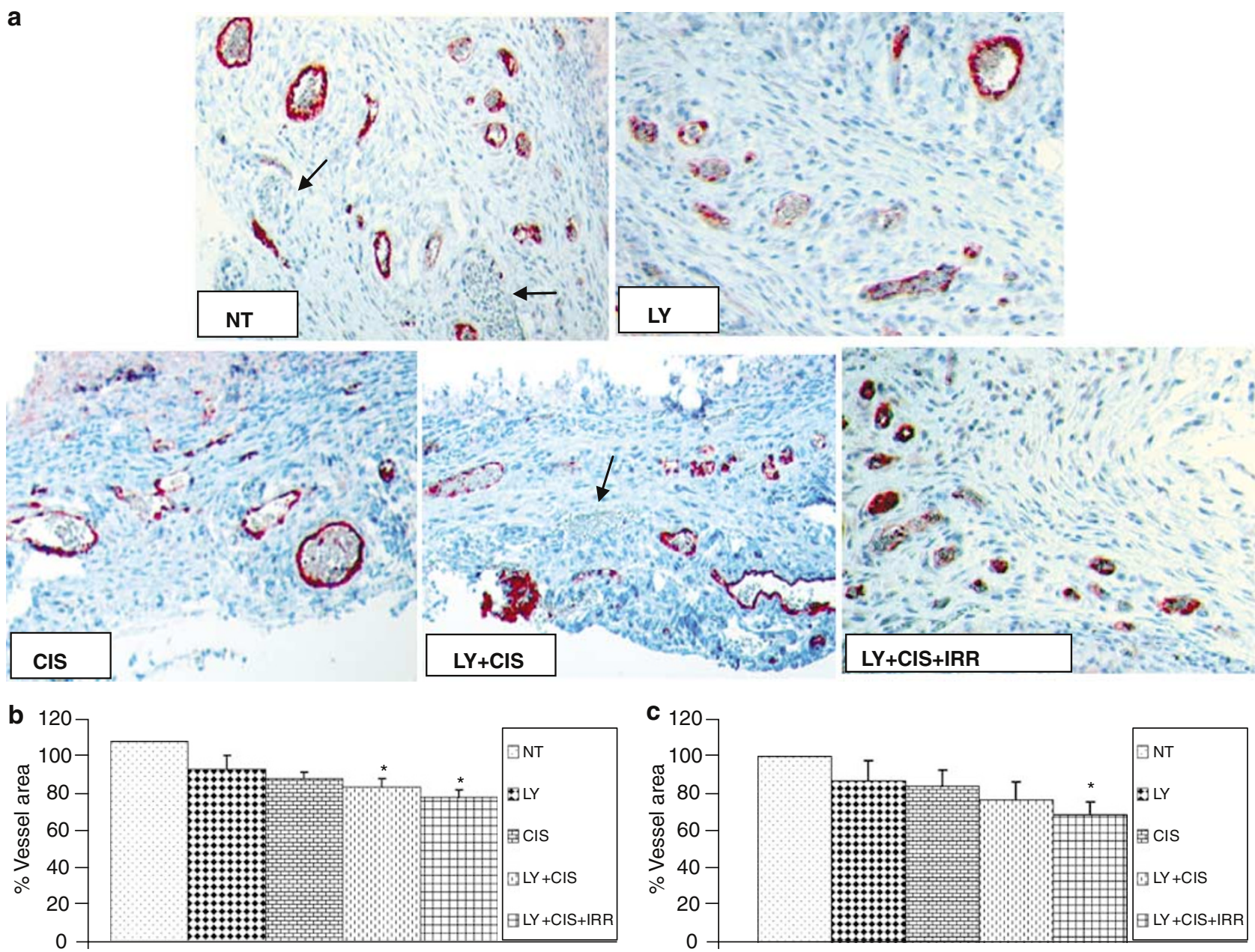

Figure 9 Combination treatment has little effect on normal angiogenesis. Paraffin-embedded scaffolds sections were stained for normal blood vessels using anti-human Von Willebrand factor antibodies. (a) Representative photomicrographs of treatment groups; notreatment (NT), LY294002 (LY), cisplatin (Cis), LY + Cis and LY + Cis + Irr. Solid arrows point to unstained vessels of mouse origin. (b) Vessel area in the tumor samples was quantified using Metamorph software and the results are expressed as \% vessel area + s.e. (c) The number of stained microvessels was counted blindly in six random high-power fields per implant at $\times 200$ magnification and the results are expressed as \% vessel count + s.e. ${ }^{*}$, Represent significant decrease $(P<0.05)$ in \% vessel area as compared to the control.

(LY294002) and cisplatin significantly enhances the therapeutic efficacy of radiation therapy by preferentially targeting tumor blood vessels.

\section{Acknowledgement}

This work was supported by NIH Grant DE 13161 (P. J. P.).

\section{References}

1 Folkman J. Tumor angiogenesis: therapeutic implications. N Engl J Med 1971;285:1182-1186.

2 Folkman J. What is the evidence that tumors are angiogenesis dependent? J Natl Cancer Inst 1990;82: 4-6.
3 Hanahan D, Folkman J. Patterns and emerging mechanisms of the angiogenic switch during tumorigenesis. Cell 1996;86:353-364.

$4 \mathrm{Xu} \mathrm{Y,} \mathrm{Liu} \mathrm{YJ,} \mathrm{Yu} \mathrm{Q.} \mathrm{Angiopoietin-3} \mathrm{inhibits} \mathrm{pulmonary}$ metastasis by inhibiting tumor angiogenesis. Cancer Res 2004;64:6119-6126.

5 Nor JE, Christensen J, Mooney DJ, et al. Vascular endothelial growth factor (VEGF)-mediated angiogenesis is associated with enhanced endothelial cell survival and induction of Bcl-2 expression. Am J Pathol 1999;154:375-384.

6 Ferrara N, Davis-Smyth T. The biology of vascular endothelial growth factor. Endocr Rev 1997;18: 4-25.

7 Neufeld G, Cohen T, Gengrinovitch S, et al. Vascular endothelial growth factor (VEGF) and its receptors. FASEB J 1999;13:9-22.

8 Gorski DH, Beckett MA, Jaskowiak NT, et al. Blockage of the vascular endothelial growth factor stress response increases the antitumor effects of ionizing radiation. Cancer Res 1999;59:3374-3378. 
9 Nor JE, Mitra RS, Sutorik MM, et al. Thrombospondin1 induces endothelial cell apoptosis and inhibits angiogenesis by activating the caspase death pathway. J Vasc Res 2000;37:209-218.

10 Shinkaruk S, Bayle M, Lain G, et al. Vascular endothelial cell growth factor (VEGF), an emerging target for cancer chemotherapy. Curr Med Chem AntiCanc Agents 2003;3:95-117.

11 Geng L, Donnelly E, McMahon G, et al. Inhibition of vascular endothelial growth factor receptor signaling leads to reversal of tumor resistance to radiotherapy. Cancer Res 2001;61:2413-2419.

12 Klement G, Baruchel S, Rak J, et al. Continuous lowdose therapy with vinblastine and VEGF receptor-2 antibody induces sustained tumor regression without overt toxicity. J Clin Invest 2000;105:R15-R24.

13 Kerbel RS, Yu J, Tran J, et al. Possible mechanisms of acquired resistance to anti-angiogenic drugs: implications for the use of combination therapy approaches. Cancer Metastasis Rev 2001;20:79-86.

14 Brem H, Gresser I, Grosfeld J, et al. The combination of antiangiogenic agents to inhibit primary tumor growth and metastasis. J Pediatr Surg 1993;28: 1253-1257.

15 Minischetti M, Vacca A, Ribatti D, et al. TNP-470 and recombinant human interferon-alpha2a inhibit angiogenesis synergistically. $\mathrm{Br} \mathrm{J}$ Haematol 2000;109: 829-837.

16 Hendry JH, West CM, Moore JV, et al. Tumour stem cells: the relevance of predictive assays for tumour control after radiotherapy. Radiother Oncol 1994;30: 11-16.

17 Paris F, Fuks Z, Kang A, et al. Endothelial apoptosis as the primary lesion initiating intestinal radiation damage in mice. Science 2001;293:293-297.

18 Garcia-Barros M, Paris F, Cordon-Cardo C, et al. Tumor response to radiotherapy regulated by endothelial cell apoptosis. Science 2003;300:1155-1159.

19 Kumar P, Miller AI, Polverini PJ. p38 MAPK mediates gamma -irradiation-induced endothelial cell apoptosis and VEGF protects endothelial cells through PI3K-Akt-Bcl-2 pathway. J Biol Chem 2004; 279:43352-43360.

20 Datta SR, Dudek H, Tao X, et al. Akt phosphorylation of BAD couples survival signals to the cell-intrinsic death machinery. Cell 1997;91:231-241.
21 Cardone MH, Roy N, Stennicke HR, et al. Regulation of cell death protease caspase-9 by phosphorylation. Science 1998;282:1318-1321.

22 Datta SR, Brunet A, Greenberg ME. Cellular survival: a play in three Akts. Genes Dev 1999;13:2905-2927.

23 Kumar P, Amin MA, Harlow LA, et al. Src and phosphatidylinositol 3-kinase mediate soluble E-selectininduced angiogenesis. Blood 2003;101:3960-3968.

24 Peters MC, Polverini PJ, Mooney DJ. Engineering vascular networks in porous polymer matrices. J Biomed Mater Res 2002;60:668-678.

25 Polverini PJ, Nor JE, Peters MC, et al. Growth of human blood vessels in severe combined immunodeficient mice. A new in vivo model system of angiogenesis Methods Mol Med 2003;78:161-177.

26 Nor JE, Christensen J, Liu J, et al. Up-regulation of Bcl2 in microvascular endothelial cells enhances intratumoral angiogenesis and accelerates tumor growth. Cancer Res 2001;61:2183-2188.

27 Viloria-Petit AM, Kerbel RS. Acquired resistance to EGFR inhibitors: mechanisms and prevention strategies. Int J Radiat Oncol Biol Phys 2004;58:914-926.

28 Humar R, Kiefer FN, Berns H, et al. Hypoxia enhances vascular cell proliferation and angiogenesis in vitro via rapamycin (mTOR)-dependent signaling. FASEB J 2002;16:771-780.

29 Sheridan MT, O’Dwyer T, Seymour CB, et al. Potential indicators of radiosensitivity in squamous cell carcinoma of the head and neck. Radiat Oncol Invest 1997;5:180-186.

30 Bernhard EJ, Stanbridge EJ, Gupta S, et al. Direct evidence for the contribution of activated N-ras and Kras oncogenes to increased intrinsic radiation resistance in human tumor cell lines. Cancer Res 2000;60 6597-6600.

31 Gupta AK, Cerniglia GJ, Mick R, et al. Radiation sensitization of human cancer cells in vivo by inhibiting the activity of PI3 K using LY294002. Int J Radiat Oncol Biol Phys 2003;56:846-853.

32 Edwards E, Geng L, Tan J, et al. Phosphatidylinositol 3kinase/Akt signaling in the response of vascular endothelium to ionizing radiation. Cancer Res 2002;62: 4671-4677.

33 McKenna WG, Muschel RJ. Targeting tumor cells by enhancing radiation sensitivity. Genes Chromosomes Cancer 2003;38:330-338. 